\title{
Capsule Endoscopy - State of the Technology and Computer Vision Tools After the First Decade
}

\author{
Michał Mackiewicz \\ Scool of Computing Sciences, University of East Anglia \\ United Kingdom
}

\section{Introduction}

Wireless Capsule Endoscopy (WCE) is a recent and exciting technology, which involves recording images of the entire Gastrointestinal (GI) tract including the parts of the human body never before seen outside operative surgery. The development of the capsule was first announced in Nature in 2000 by Iddan et al. (2000). Since then a number of different capsules have been launched by different vendors which varied slightly in their purpose, but retained the principle of wireless non-invasive investigation of the GI tract. It is particularly suited for computer-assisted diagnosis, as it records a large quantity of data (mostly, but not exclusively images) from the human gut, which consequently requires a time-consuming visual assessment that can be carried out only by an experienced clinician. The duration of this assessment, which involves the scrutiny of a video comprising approximately 50, 000 frames, varies between one to two hours. Thus, it can be seen that in terms of time requirement, the WCE is a very costly medical imaging procedure. This opens a door for computers to aid the analysis of the WCE footage, by reducing the time required to reach the diagnosis and thus the cost of the procedure, making it a more affordable technique. This view is supported by the leading endoscopists in the United Kingdom:

"The cumbersome analysis of WCE images has been the major faction in preventing the spread of WCE to become a routine service in every DGH." Dr Jonathan Green, Secretary of the Endoscopy Committee of the British Society of Gastroenterologists.

"The key constraint for uptake of WCE is the time taken to read and report the examination. Increased automation of reading of WCE will have a dramatic effect on the uptake of this important endoscopic technique, and to the costs of providing the service." Dr Roland Valori, National Endoscopy Lead.

Another aim of computer-assisted WCE video analysis can be considered in terms of improvement of the clinicians diagnosis. Here, the computer assumes the role of an expert system able to give a second opinion on the state of the patient.

This chapter reviews the problems of Wireless Capsule Endoscopy video analysis from an image processing and pattern recognition perspective. As it has been ten years since the first capsule was launched we consider there is a need for a review of the work to date which would sum up the current state-of-the-art and draw certain conclusions about the future 
directions of the research. Moreveor, we review in detail the technologies of the capsule as they were developing.

The paper is organised in the following manner. In Section 2, WCE technology and its clinical use are described. In Section 3, a review of the different types of the computer vision algorithms is given. Finally, Section 4, contains the conclusions.

\section{Technology and its clinical use}

The first wireless capsule endoscope was launched in 2001 by Given Imaging Ltd, and reported in an article in "Nature" Iddan et al. (2000). Since the device received FDA (American Food \& Drug Administration) clearance in August 2001, over 1,000,000 examinations have been conducted globally. The $11 \mathrm{~mm}$ x $26 \mathrm{~mm}$ M2A capsule (later rebranded PillCam SB (SB stands for small bowel)) (see Figures 1 and 2) is propelled passively, hence having been swallowed, it is propelled through the food tract by normal peristaltic movement of the human gastrointestinal (GI) system, usually reaching the colon, before being expelled naturally from the body. One end of the capsule contains an optical dome with six white Light Emitting Diodes (LEDs) and a CMOS camera that captures 2 images (circular shape from a square of $256 \times 256$ pixels) a second. These images are compressed using JPEG and relayed via a transmitter using a radio frequency signal to an array of aerials, which are attached to the patient's body, from where they are transferred over the wires to a data-recorder worn by the patient on a belt. The sensor array allows for continues triangulation of the position of the capsule inside the body of the patient so that the trajectory of the capsule passage can be later displayed on the workstation monitor. The accuracy of the capsule location provided by this method was reported to be $\pm 3 \mathrm{~cm}$ by Ravens \& Swain (2002). After 8 hours (the capsule two silver-oxide batteries lifetime), the data-recorder is removed and the image data uploaded to a workstation for viewing and analysis. The upload process originally took two to three hours using early versions of the Given Imaging Rapid Reader (RR) software, but a more recent version of this application has reduced this to around 30 minutes. The stored data consists of $\sim 50,000$ images, and is viewed as a video sequence using software provided by the manufacturers (RR) (see Figure 3)).

In 2007 PillCam SB 2 was cleared for marketing in the US. According to the manufacturers, it offers advanced optics and a wider field of view. PillCam SB 2 also captures nearly twice the mucosal area per image. It also provides Automatic Light Control for optimal illumination of each image.

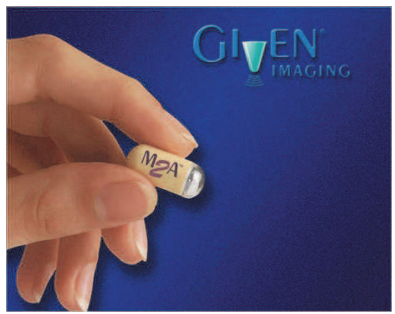

Fig. 1. M2A capsule

The clinical procedure is a simple and painless process, which is one of the factors why the patients prefer WCE to conventional endoscopy methods Melmed \& Lo (2005). Before the procedure begins, patients must fast overnight. Moreover, some studies suggest that results 


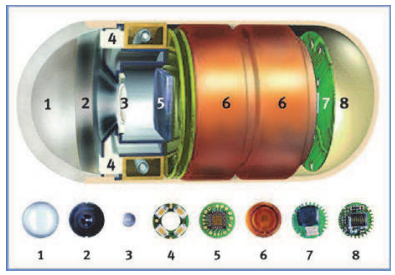

Fig. 2. The inside of the M2A capsule - 1) Optical dome, 2) Lens holder, 3) Lens, 4) LEDs, 5) CMOS imager, 6) Battery, 7) RF transmitter, 8) Antenna.

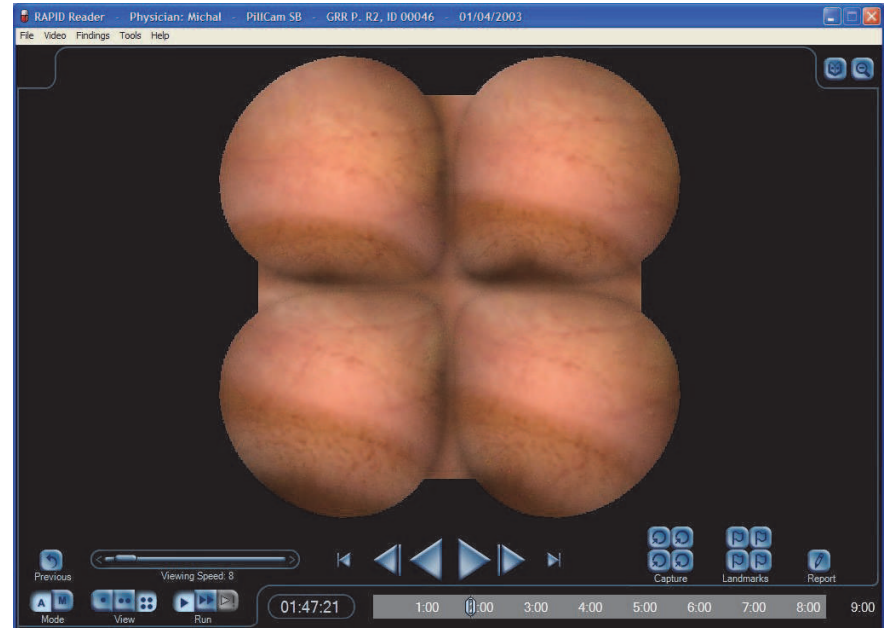

Fig. 3. Rapid Reader ver.4 software - A part of the Given Imaging diagnostic system. Note, the quad view - four images blended on the edges and displayed at the same time

may be improved by bowel preparation i.e. ingesting a drug that shortens food and capsule transit times through the GI track Dai et al. (2005). The exam begins by the attachment of the antennas to the patient's chest, which are then connected to the data-recorder worn on a belt. The capsule starts acquiring images and transmitting them immediately after it is removed from its magnetic holder. After a brief capsule test by the physician (less than one minute), it should be immediately ingested by the patient. Water and food intake can begin after 2 and 4 hours respectively. Patients are asked to monitor a blinking diode on the belt pack, which indicates the quality of the signal reception. The patient should not exercise during the procedure, and avoid any powerful electromagnetic field source.

In October 2005, Olympus launched a competitor system called EndoCapsule (see Figure 4) in Europe. Their device, acquires images at the same rate and at the same resolution as the Given Imaging PillCam. The difference lies in the use of a different imaging technology CCD, which the manufacturers claim is of higher quality Fuyono (2005). Another feature of the EndoCapsule is the Automatic Brightness Control (ABC), which was applied from the traditional endoscope technology. $\mathrm{ABC}$ provides an automatic illumination adjustment as the conditions in the GI track vary. EndoCapsule also features a real-time viewing device (see Figure 5), which allows the physician to watch the capsule examination in real time. Moreover, 
the EndoView software also features image pre-processing capabilities, which result in a high image quality.

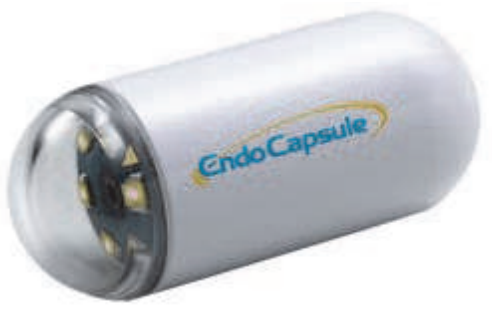

Fig. 4. Olympus EndoCapsule

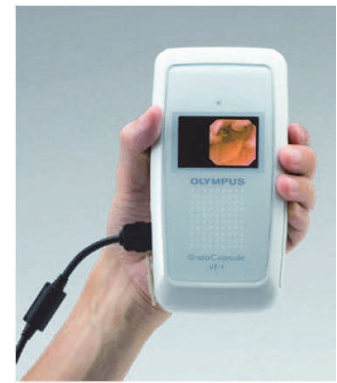

Fig. 5. Olympus Real-time viewer (taken from Olympus website)

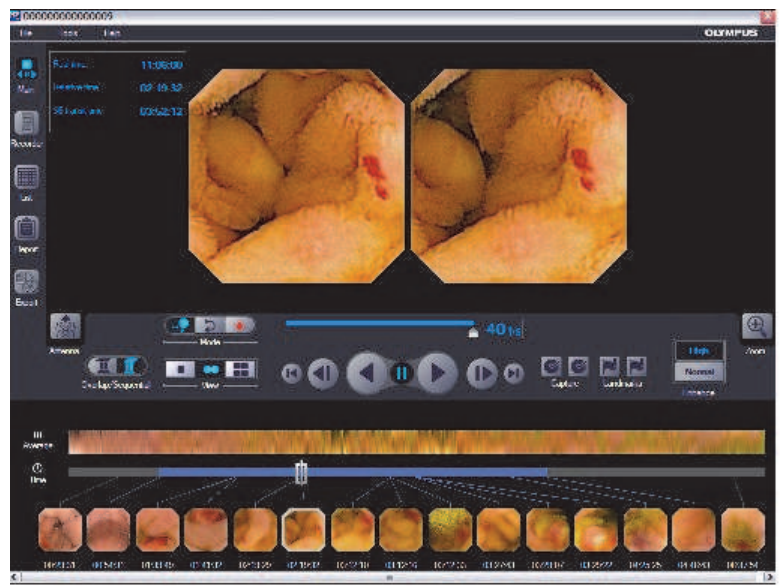

Fig. 6. Olympus EndoView video viewing software

In December 2004, FDA approved a second type of capsule developed by Given Imaging the PillCam ESO, which allows the evaluation of oesophageal disease. The motivation for developing this capsule lay in the rapid capsule transit through the oesophagus. Here the frame rate of only two frames per second (of the original PillCam SB) did not provide enough data for a thorough examination of this GI region to be undertaken. This opinion was also 


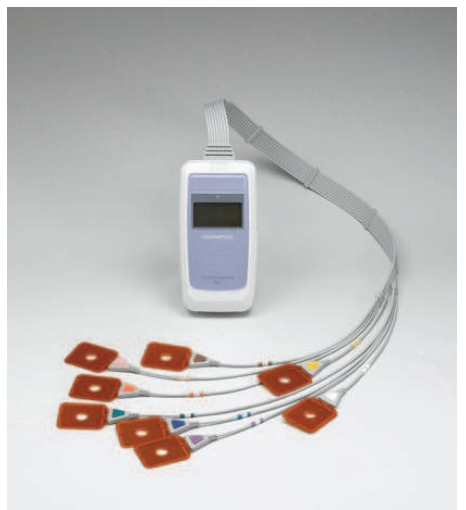

Fig. 7. Olympus EndoCapsule sensor array and data recorder (taken from Olympus website)

reported in Neu et al. (2003), where the authors concluded that esophageal capsule endoscopy could not be achieved without further technical developments. The response to this demand materialised in the development of the PillCam ESO which has the higher frame rate and CMOS cameras positioned at both ends of the capsule. This capsule acquires and transmits seven frames per second from each camera, giving a total of 14 frames per second Mishkin et al. (2006). Due to the increased frame rate, the capsule battery life is only 20 minutes, which is ample time for the capsule to visualise the entire oesophagus.

In October 2006, Given Imaging received the CE Mark to market a third capsule - the PillCam COLON throughout the European Union. This device was developed as a diagnostic test to visualize the colon. The capsule measures $11 \mathrm{~mm}$ by $31 \mathrm{~mm}$ - slightly larger than the previous two capsules. Similar to PillCam ESO, the capsule has cameras at both ends. These capture 4 images a second for up to 10 hours. A new feature in Given Imaging capsules is an automatic lighting control. Since the lumen of the colon is wider than the small bowel and also highly compartmentalised, the PillCam COLON capsule optics captures more than twice the coverage area and depth of field of the PillCam SB capsule. After switching on the capsule and within a few minutes of image transmission, the device enters a delay mode. This lasts approximately 2 hours, after which it "wakes" up to resume the image transmission. This saves the battery power during the transit of the capsule through the earlier parts of the gut, allowing longer transmission from the relevant GI region - the colon. In 2009, the second-generation capsule, PillCam COLON 2, was cleared by the European Union. PillCam COLON 2 offers additional features such as: intelligent functionality, superior imaging and a convenient workflow. The capsule has the ability to adjust the frame rate in real time to maximize colon tissue coverage. The imaging devices on either end of the capsule provide a near $360^{\circ}$ view of the colon. The manufacturers claim that with this capsule the patient study process has been simplified and hence it allows for more efficient utilisation of staff time and resources.

Another competitor capsule was launched by the IntroMedic IntroMedic (n.d.). Their capsule known as MicroCam received the CE Mark in 2007. The dimensions of the capsule are $11 \times 24 \mathrm{~mm}$ and the $150^{\circ}$ field of view. During the $11 \mathrm{~h}$ battery life time, the capsule is capable of capturing around 120,000 images with a frame rate of 3 images per second and a resolution of $320 \times 320$ pixels. The manufacturers also supply a real-viewing device. The 
MiroView software contains features such as: Capsule Positioning, Suspected GI Bleeding, Image Enhancement, Multi-Display Viewing and the Quick View Mode.

Yet another capsule has been developed in China. The OMOM capsule Liao et al. (2008) has been also FDA approved and received the CE Mark. The novel features offered by OMOM include the first two-way transmission allowing the image capture rate and the light level adjustment; and the vest jacket containing the RF sensors.

\subsection{Future development directions}

All active endoscopes (those whose movement can be controlled) produced so far are wired ie. they transmit the images from the camera to the display over a wire. The capsules described in this chapter are all passive, which means they are propelled by peristalsis and their movement cannot be controlled. An active propulsion system would offer obvious benefits. It is not surprising, therefore, that it is the focus of intensive research. Two main approaches in this field have been proposed: internal, where the locomotion results from the mechanisms contained in the capsule and external, which utilises forces transmitted from the outside, usually by the means of the magnetic field Toennies et al. (2010). Below, we will describe a few developments in these fields that were reported in the literature.

In Sendoh et al. (2003), the authors propose an external type of propelling the capsule using a magnetic accurator. The device uses a permanent magnet in the capsule and an external rotational magnetic field to control the capsule movement. The authors report a preliminary experiment in which they used a dummy capsule without any endoscopic functions. Kosa et al. (2008) propose a miniature swimming mechanism that uses MRI's magnetic fields for powering the capsule. Their method uses both the static and radio frequency (RF) magnetic fields available in MRI to generate propulsion force and deliver wireless energy. Another magnetic propulsion mechanisms have been proposed by Gao et al. (2010).

Olympus has been also working on the development of a new generation capsule endoscope, which features magnetic propulsion Olympus News Release (2004) (see Figure 8). Apart from the novel propulsion and guidance system, the capsule designers aim to provide provide a drug delivery system, which would administer drugs directly to an affected area; a body fluid sampling system, taking body fluid extracts for diagnosis and analysis; and also an ultrasound scan capability.

RF System Lab Company RF Systems (n.d.) has also announced the intention of producing a Norika capsule with a magnetic field based propulsion (see Figure 9). In December 2005, they also announced the design of the new Sayaka capsule, which would have a lens on the lateral surface of the capsule instead of the front as in the capsules of the competitors (see Figure 10). The inventors claim that such a design would obtain clear-cut images of the gastrointestinal wall while the capsule spins in the GI tract with the stepping angle of $7.5^{\circ}$. Sayaka acquires images at a rate of 30 frames per second, which generates $\sim 870,000$ over an eight hour period of operation. The images are later combined together in a process called mosaicing, which produces an image (map) of the GI tract walls.

Further applications of magnetic fields are presented by Lenaerts \& Puers (2006), where the authors propose to use an inductive link to power a wireless capsule endoscope. The capsule, known as the Intracorporeal Videoprobe (IVP2, see Figure 11) Arena et al. (2005) is induction-powered and equipped with a tilting image sensor on a motorised plate (see Figure 12) and a telemetric datalink. In Turgis \& Puers (2004), the authors describe the video compression method of this system. According to Innovative imaging probes for endoscopy (2005) 
, the capsule is still a long way from becoming a commercial product. Moreover, the author could not find any publication on this capsule in the last four years.

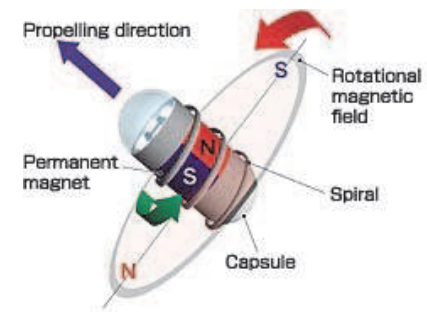

Fig. 8. Conceptual diagram of the future Olympus capsule guidance principle (taken from Olympus New Release)

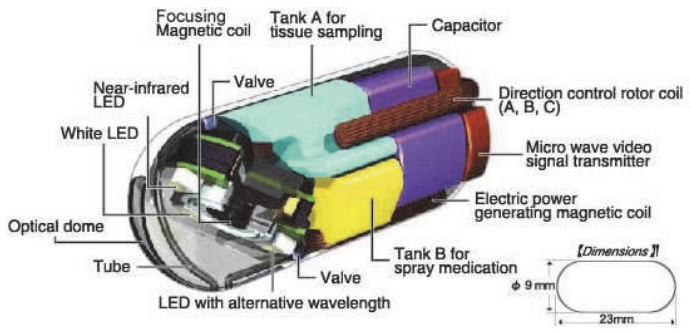

Fig. 9. Norika capsule design (taken from RF System website)

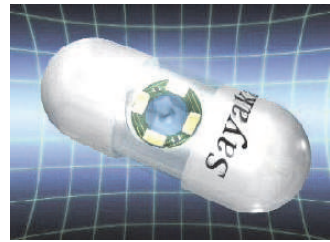

Fig. 10. Sayaka capsule (taken from RF System website)

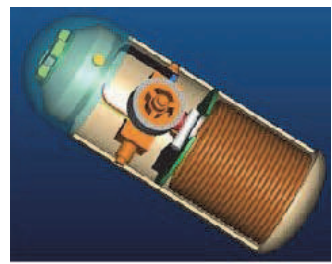

Fig. 11. IVP2 capsule (taken from IVP project website)

An internal approach to capsule locomotion has been proposed by Quirini et al. (2007). The authors presented the design of the locomotion for a 12-legged, swallowable (11 mm diameter by $25 \mathrm{~mm}$ long), endoscopic capsule to be utilised in the lower GI tract (large bowel). The capsule utilises a slot-follower mechanism driven via lead-screw generating $2 / 3 \mathrm{~N}$ of force at each leg tip. 


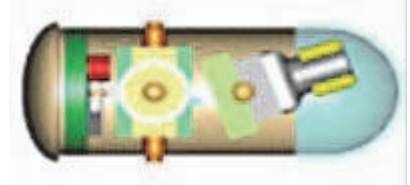

Fig. 12. IVP2 capsule steering system (taken from IVP project website)

\subsection{WCE clinical importance}

The gastrointestinal (GI) tract consists of the oesophagus, stomach and duodenum (upper GI tract), the jejunum, ileum (small bowel), colon and rectum.

Fibre optic gastrointestinal endoscopy, introduced in the early 1970s enabled effective diagnosis and biopsy of disease in the lumen of the stomach and duodenum (gastroduodenoscopy OGD) and shortly afterwards the colon and rectum (colonoscopy). In the 1980s, videoendoscopy using microchip cameras improved image resolution and the ease of use of the equipment.

These innovations enabled clinicians to diagnose a large number of GI pathologies which occur mostly in the stomach, duodenum and colon. Examination of the remainder of the small intestine using a conventional endoscopy (the jejunum and ileum) was until very recently limited only to the first few centimeters of jejunum and last few centimeters of the terminal ileum. However, this part of the GI tract can be the site of "obscure" bleeding, inflammation and a rare location for tumours. There exist non-standard endoscopy methods to investigate this part of the small bowel. However, they use a flexible tube to propel the endoscope along the bowel, which given the length of the investigated gut (up to $4.5 \mathrm{~m}$ ) and its shape with many loops, rendered the procedure very difficult and uncomfortable to patients. Other means of imaging the small bowel, such as computerised tomography (CT) or magnetic resonance (MR) axial imaging cannot provide a direct view of the tissue. Hence, there was a clinical need to provide a tool which would enable clinicians to diagnose the jejunum and the ileum more efficiently.

The wireless capsule endoscope (WCE) developed by Given Imaging Ltd was an answer to this demand, which for the first time allowed an endoscope to record the high-resolution images of its full passage through the GI tract including the parts of the human body never before seen outside operative surgery.

Following many clinical studies Gay et al. (2004); Leighton et al. (2006); Selby (2004); Viazis et al. (2005) into the efficacy of WCE in clinical practice, the superior imaging capabilities of WCE above contrast imaging and CT imaging became clear. These studies concentrated on patients with "obscure" GI bleeding; those who had lost blood into the lumen with no cause found at OGD or colonoscopy. Consequently, WCE is now established as the first line diagnostic test for patients with this condition, and received approval as such from the National Institute for Clinical Excellence (NICE) in the UK in 2004.

WCE also received attention with regard to the diagnosis and assessment of patients with suspected or known Crohn's disease (CD) Bona et al. (2006); Dubcenco et al. (2005); Pennazio (2004a;b); Swain (2005), a chronic inflammatory disease affecting any part of the GI tract, but most often localised in the terminal ileum. This disease is of increasing prevalence in the developed world Bernstein (2006), and causes significant morbidity to patients. It is difficult to diagnose, partly due in the lack of sensitivity of contrast radiology to minor mucosal pathologies. WCE offers significant improvements to the diagnosis of CD due to its greater sensitivity, and also allows follow-up examinations without exposing the patient to 
the hazards of ionising radiation. The capsule has also been found useful in the diagnosis of another form of small bowel inflammation - Celiac disease Culliford et al. (2005).

Cancer of the small bowel is rare but often has a high mortality rate since it tends to be diagnosed late. A number of clinical studies Cobrin et al. (2006); Urbain et al. (2006); van Tuyl et al. (2006) have found WCE as a sensitive tool in detecting cancerous conditions. Therefore, if employed with this group of patients, capsule endoscopy is expected to offer a clinical benefit in terms of cancer survival rate. Peutz-Jegher disease is a hereditary condition that involves the formation of small bowel polyps with a potential to become malignant, especially when they reach a large size. In Brown et al. (2006), the authors suggest that wireless capsule endoscopy should become the investigation of choice in those undergoing follow-up in interval surveillance for this condition.

Patients with obscure diarrhoea abdominal pain and possible functional bowel disease have also been examined in series of studies to assess potential diagnostic benefits of WCE, but in this heterogeneous group no clear benefit has been demonstrated to date Fry et al. (2006). The capsule has also proven useful in studies concerning the impact of drugs on the gastrointestinal tract Qureshi (2004). Moreover, children can benefit from the device as well as adults Argüellas-Arias et al. (2004).

The development of PillCam ESO with a faster frame acquisition rate has allowed the non-invasive imaging of oesophageal conditions in selected groups of patients. This safe and acceptable-to-patients form of WCE may represent an alternative to conventional upper endoscopy, and give a clinical benefit in the assessment of endoscopic signs of esophageal varices and portal hypertension Caunedo-Alvarez \& Herrerias-Gutierrez (2006); Eisen et al. (2006); Eliakim et al. (2004). Early studies from the PillCam COLON Eliakim et al. (2006); Schoofs et al. (2006) launched for clinical use by Given Imaging in 2006 show promising accuracy compared with colonoscopy and suggest further clinical trials to establish the efficacy and appropriate role for this application of capsule endoscopy.

It is believed by some researchers Iddan et al. (2000) that WCE approximates 'physiological endoscopy' as it is more closely aligned with human physiology than conventional endoscopy, where sedation and air insufflation are required to provide a clear passage for the conventional wired scope. These factors result in the distortion of the natural appearance of the viewed area in traditional endoscopy and colonoscopy.

\section{Computer vision tools in WCE video analysis}

In this section, we give a detailed review of the computer vision tools that were either developed by the manufacturers or reported in the scientific literature. This section was divided into the main areas of research, namely: topographic video segmentation (Section 3.1), bleeding detection (Section 3.2), abnormality detection (Section 3.3), capsule retention detection (Section 3.4), adaptive viewing speed adjustment(Section 3.5), non-informative frame filtering (Section 3.6) and intestinal contraction detection (Section 3.7).

\subsection{Topographic video segmentation}

The GI tract consists of the mouth, oesophagus, stomach, small intestine and large intestine (colon). Once switched on, the capsule endoscope is usually outside the body of the patient for no more than a few seconds, before it is placed in the mouth, where it usually stays for a few seconds, before it is swallowed. After which, it is pushed by peristalsis along the oesophagus, in which it stays for around two seconds. Hence, usually we can see only around 3-5 images 
taken inside the oesophagus (the capsule transmits two images per second), before the capsule reaches the esogastric junction (EJ) separating the oesophagus from the stomach. Typically, the time spent in the stomach (GTT) is around 15 minutes. However, this time can be significantly longer, and it is possible for a capsule to stay in the stomach for several hours before it passes through the pylorus (the valve between the stomach and the intestine). The small intestine is the longest part of the GI tract, and the capsule usually spends around four hours in transit through this region. It ends with the ileocaecal valve (IV), which marks the beginning of the colon.

Finding the pylorus in the video can be difficult and time-consuming, even for an experienced viewer, as visually the stomach tissue in the pyloric region and the tissue at the beginning of the intestine appear very similar. Annotating the place in the video where the capsule enters the IV is even more difficult, since intestine and colon tissues are very similar and are often contaminated with faecal material that occludes the camera view. Annotating the EJ is relatively the easiest of these three tasks, as the physical features inside the mouth, oesophagus and stomach are visually quite unique and, thus significantly different.

The possibility of classifying different organs was first indicated in 2004 by Berens et al. (2004) who reported building a Stomach/Intestine classifier which could be used to predict the pylorus. Later, Berens, Mackiewicz \& Bell (2005) propose an extended discriminator that could also determine the Ileocecal Valve (IV). In that paper, they also compare the performance of two classifiers used to build the discriminators: k-nearest neighbour $(k N N)$ and Support Vector Classifier (SVC). Next, Berens, Mackiewicz, Fisher \& Bell (2005) present a further discriminator for the Mouth/Oesophagus\&Stomach regions and then combine all the discriminators to provide an estimate of the time taken for the capsule to pass through the stomach ( known as the Gastric Transit Time (GTT)) and the intestine (known as the Intestinal Transit Time (ITT)). These are used by clinicians as important indicators of the health of the digestive system. The discriminators utilise hue saturation histograms which are compressed using a hybrid transform, incorporating both the Discrete Cosine Transform (DCT) and Principal Component Analysis. More recently, Mackiewicz, Berens \& Fisher (2008) expand their previous work on capsule tissue discrimination and present yet another WCE video segmentation algorithm based on a Hidden Markov Model (HMM). They compare the performance of two classifiers Multivariate Gaussian and SVC, which were built within the framework of HMM. They also show that adding texture and motion features in the classifiers improves the results. Moreover, they note that WCE images often contain artifacts and shadows that can obscure the underlying tissue images. To address these problems, they develop a technique for extracting only those image regions that contain clearly visible tissue. They show that combining features for these regions with features derived from the entire image provides more accurate discrimination between the GI body parts.

An extensive work on the same topic was also carried out by another research group in Portugal starting with Coimbra et al. (2005), where they present an attempt to segment a WCE video into meaningful parts. They divide the video into four zones: Entrance (Z1) - consisting of image frames acquired from the mouth and oesophagus as well as those acquired before the capsule is swallowed; Stomach (Z2) - whose limits are determined by the oesogastric junction and the pylorus; small intestine (Z3) - delimited by the pylorus and IV; Colon (Z4) - from IV to the end of the footage. MPEG-7 descriptors (Scalable Colour and Homogeneous Texture) are used as low-level image features. The classification is performed using a Bayesian classifier, which assigns a topographic location label to each frame in the video. Iteration is used to minimise the segmentation error, resulting in three parameters that 
show the positions of transitions between the four previously defined zones. Later Coimbra, Campos \& Cunha (2006b) show that using a Support Vector Classifier instead of the Bayesian approach significantly improves the results, which can be used to estimate the capsule Gastric and Intestinal Transit Times. The authors have later shown that combining content with context features may give an additional boost to WCE video segmentation Coimbra, Kustra, Cunha \& Campos (2006). Contextual features may include spatial location of the capsule inside the body of the patient and capsule displacement velocity. The authors conclude that such an approach mimics more closely additional expert knowledge that the clinician draws on in order to perform the annotation more accurately.

More recently, Duda, Zielinski, Fraczek, Bulat \& Duplaga (2007) report the results of a study in which they attempt to classify the images from the upper part of the GI tract into a larger number of distinctive regions than it was done before. They choose six anatomical regions: A) oesophagus, B) cardia, C) fundus, D) corpus of the stomach, E) pylorus and F) duodenal cap, whereas the previous studies looked only at oesophagus, stomach and small intestine. As in their other work Duda, Zielinski, Duplaga, Grega \& Leszczuk (2007), they use MPEG-7 features together with Vector Quantisation and Principal Component Analysis to produce the feature vectors; and Neural Networks as the classifiers. The study is small size and it utilises only two video sequences. The authors report only the classification results and do not attempt to segment the actual videos.

Lee et al. (2007) present yet another method for detecting GI organ boundaries. In their work, they try to segment the CE video into 5 regions: oesophagus; stomach; duodenum and jejunum; ileum; and colon using frequency domain functions derived from the variations of the intensity signal across the video. The idea for their algorithm is based on the fact that each digestive organ has different patterns of intestinal contractions. These patterns have been earlier used in the analysis of biogenic signals e.g. Electrogastrogram, which is a non-invasive recording of the stomach electrical activity. The analysis of the aforementioned frequency functions leads to the event boundaries which indicate either entrance to the consecutive organ or unusual events in the same organ, such as intestinal juices, bleeding, ulceration, and unusual capsule movements. These events are then further classified and merged into higher level events that represent digestive organs which leads to a tree-like representation of the $\mathrm{CE}$ events. The authors report that the proposed scheme can detect the most of stomach and duodenum correctly, but the performance on ileum and cecum is worse. Similar findings regarding difficulties in locating the IV were reported in the other studies that looked only at colour and texture features Coimbra, Campos \& Cunha (2006b); Mackiewicz, Berens \& Fisher (2008).

\subsection{Bleeding detection}

Rapid reader - the Given proprietary software provided with the PillCam capsule, has an automatic image analysis tool called Suspected Blood Indicator (SBI). This tool however, was reported to have a very low specificity and sensitivity Signorelli et al. (2005) and as authors suggest, it can be used only as a complementary rapid screening tool, which cannot replace the complete view of the recordings by the specialist. Moreover, it is not obvious how SBI exactly detects red areas since Given Imaging has not revealed this. Similarly, we do not know how Olympus EndoCapsule red spot detector works. In Signorelli et al. (2005), the authors state that in their observations the ability of SBI to detect red lesions was related neither to their size nor to the length of the lesion event (number of frames). It is believed that as the new 
versions of the Rapid Reader software are released, the SBI algorithm is improved, however the author of this chapter has no knowledge of how significant if any these improvements are. Hwang et al. (2006) propose an algorithm that as they claim can detect bleeding areas in the capsule videos. The algorithm uses Expectation Maximization (EM) clustering and Bayesian Information Criterion (BIC). The authors manually segmented around 200 images into blood and non-blood regions. Then, they selected 16,000 bleeding and 45,000 non-bleeding pixels and modelled the colour distribution of these regions using Gaussian mixtures in $R G B$ colour space. Bayesian Information Criterion was used to decide the number of clusters. In the first step of the algorithm, dark pixels are removed. In the second step, the algorithm chooses those pixels $x$ to be bleeding candidates for which conditional probability $p$ ( $x \mid$ bleeding) of a pixel $x$ given by bleeding pixels is significantly larger than conditional probability $p(x \mid$ non - bleeding $)$ of a pixel $x$ given by non-bleeding pixels; and also it is larger than a certain predefined threshold. In the final step of the algorithm, the areas of bleeding regions are calculated and all segmented regions containing less than 1,000 pixels are rejected. To test the results of bleeding detection, the authors selected 15,222 capsule images of which 1,731 contained blood from three different videos. On this test set, a reported specificity and sensitivity were $98.10 \%$ and $92.55 \%$ respectively. Unfortunately, this algorithm was not tested on full-length video sequences, which makes it difficult to state whether it performs better than SBI.

Contrary to Hwang et al. (2006), where the authors use parametric bleeding colour distribution modelling, Mackiewicz, Fisher \& Jamieson (2008) choose a histogram based method. They claim that this was motivated by the need of fast model adaptation, which is easier in the non-parametric method. Their method uses in its first stage Hue-Saturation-Intensity colour histograms to track a moving background and bleeding colour distributions over time. Such an approach addresses the problem caused by drastic changes in blood colour distribution that occur when it is altered by gastrointestinal fluids and allow detection of other red lesions, which although are usually "less red" than fresh bleeding, they can still be detected when the difference between their colour distributions and the background is large enough. In the second stage of their method, their algorithm analyses all candidate blood frames, by extracting colour (HSI) and texture (LBP) features from the suspicious image regions (obtained in the first stage) and their neighbourhoods and classifying them using Support Vector Classifier into Bleeding, Lesion and Normal classes. They show that their algorithm compares favourably with the SBI on the test set of 84 full length videos.

Recently, Li \& Meng (2009) propose a method of bleeding and ulceration detection by means of chromaticity moments constructed from the Tchebichef polynomials. The authors divide the circular CE image into a grid of 36 non-overlapping blocks $(30 \times 30$ pixels)(similar grid was also used for feature extraction by Mackiewicz, Berens \& Fisher (2008)), from which they calculate six chromaticity moments. Next, they performed an experiment in which 5400 (1800 normal, 1800 bleeding and 1800 ulceration) image blocks were selected from 300 non-consecutive CE images extracted from 10 patient video sequences. The blocks were randomised and classified using an MLP Neural Network with 4-fold cross-validation. Finally, the authors reported sensitivity and specificity figures obtained from the block classifications. This study should be considered as preliminary as it was not performed on the full length videos. Moreover, one can note that the training and the test sets were allowed to contain the blocks from the same patient sequence as the 4 folds of the dataset were chosen after the blocks extracted from the 10 video sequences were randomised. 


\subsection{Abnormal tissue detection}

One of the first image processing studies in capsule endoscopy was conducted by Boulougoura et al. (2004) who describe an intelligent system, which they claim is capable of discriminating between normal and abnormal tissue in M2A WCE images. They use 54 feature vector elements, which are nine measures (standard deviation, variance, skew, kurtosis, entropy, energy, inverse different moment, contrast and covariance), calculated from histograms ( $1^{\text {st }}$ order statistics) of six channels $(R, G, B, H, S, V)$. The images are classified using an advanced neural network scheme containing the fusion of multiple classifiers dedicated to specific feature parameters. The authors report a detection accuracy of $100 \%$. However, this result was evaluated using only 73 capsule images ( 33 abnormal and 38 normal), which were split into the training set (23 abnormal,25 abnormal) and the test set. The size of data used in this study is insufficient to draw conclusions whether the system can be used in a working application. The authors intended to test their algorithm on the images acquired from the IVP capsule Arena et al. (2005), which was under development at that point in time.

More recently Szczypinski \& Klepaczko (2009) proposed a method of feature selection which selects relevant features by searching for a subspace that would encapsulate all vectors from one chosen pathology type by a convex hull and hence they call their algorithm Vector Supported Convex Hull (VSCH). The authors work with a high dimensional dataset extracted using MaZda software consisting of several hundred colour and texture features. The motivation for this approach was to minimise the rate of false negative errors. Apart from identifying significant features, the method also determines a classification rule based on the mathematical definition of the optimal convex hull. The authors compare their method to the Radial Basis Function network and conclude that it compares favourably with the RBF and that it ensures the desired behaviour of low false negative ratio without any explicit weighting of error types. The study should be considered as preliminary as it was carried out on a dataset extracted from only 50 images which came from 3 video sequences.

Bejakovic et al. (2009) present a method that uses color, texture and edge features to analyse the Crohn's disease lesion in CE images. They used 3 MPEG-7 visual descriptors (dominant color (DCD), homogeneous texture (HTD) and edge histogram (EHD)); and Haralick texture features calculated from 1 pixel co-occurrence matrices. They used Support Vector Machines to classify CE images into 3 classes: lesion, normal tissue, and extraneous matter (food, bile, stool, air bubbles, etc). The database used to evaluate their method contained a number of 100 frame video sequences extracted from 10 patients which were pre-annotated by the clinicians. Ten-fold patient specific cross validation was performed. The results are given as Accuracy and Recall rates for the three earlier defined classes and one of the earlier defined descriptors. Authors conclude that it is the colour which carries the most useful information with regard to the CE image classification, which confirms earlier findings by Coimbra, Campos \& Cunha (2006a).

Recently, Li et al. (2011) presented a study with an aim to develop a computer aided system to diagnose small bowel tumours. They propose a textural feature that is built on wavelet and local binary pattern. With regard to the classifier, they employ a classifier ensemble consisting of k-nearest-neighbor, multilayer perceptron neural network and support vector machine. Results obtained from the analyses of 1200 (600 normal and 600 abnormal) capsule images showed the promising performance for small bowel tumour detection. 


\subsection{Capsule retention detection}

Szczypinski et al. (2004) attempt to localise the areas in the GI tract that might be affected by Crohn's Disease (CD). In their work a Model of Deformable Rings (MDR) is used to locate the areas where the capsule moves more slowly or stops. This, according to some researchers (Tang et al., 2003) may signify the appearance of CD. The model aims to determine the movement of a tube-like surface (GI tract) by comparing adjacent video frames with regard to the displacement of its distinctive portions. MDR calculates a 2D map of the internal surface and provides an estimate of the capsule velocity. The map can be used as a quick reference, supporting identification of the segments of the GI tract and according to the authors claim, may be useful in identification of large scale pathologies.

\subsection{Adaptive viewing speed adjustment}

The main motivation for applying computer vision techniques to WCE video analysis is the potential improvement gained by reducing the overall time needed to review the data, by alerting the expert to clinically significant video frames. This may be achieved not only by automatic detection of events or segmenting the video into some meaningful parts, but also by adjusting the replay speed (number of frames displayed per second). Hai et al. (2006) propose such a method of handling the frame rate in a capsule image sequence. In their solution, instead of letting the clinician adjust the frame rate manually, video speed is adjusted by an algorithm, which plays the video at high speed in stable regions and at slower speed where significant changes between frames occur, signifying the possibility of pathologies. The authors divide each frame into 64 blocks and measure the similarity of colours between respective blocks in consecutive frames. $R G B$ histograms quantised to $16^{3}$ bins are used to describe each image block. The distance between local histograms is computed using the $L_{1}$ norm, which is later used to calculate the similarity between two frames. Moreover, the maximum and minimum of distances between blocks are collected. In addition to these colour features, the algorithm estimates motion displacement by extracting features using the KLT algorithm and tracking them using Newton-Raphson iterations. Then, these motion and similarity features are used to classify each frame by the decision tree into four states: 1) capsule and small intestine are stationary; 2) movement of the small intestine is small;3) the small intestine has larger movements and finally 4) the small intestine has abrupt changes. From the sequence of states, the delay time between consecutive frames is calculated using parametric functions, which take into account: the state to which the frame belongs, motion and similarity features, the skill of the clinician and the hardware limitations. The authors conclude that using their method the viewing time may be reduced from $2 \mathrm{~h}$ to around 30 minutes without loss of information.

The software supplied by both Given Imaging (Rapid Reader) and Olympus (EndoView) also include play speed control. Unfortunately, the details of these algorithms remain unknown. Moreover, in the more recent versions of Given's Rapid Reader, the clinician is given an option of watching a video in either "Normal Mode" or in the "Quick View Mode". Although the "Quick View" mechanism is not precisely explained in the documentation, we noticed that it uses an approach similar to that described above to reduce the viewing time of the video. It must be added though, that the "Quick View" mode skips some frames, displaying only the most suspicious (at least to the algorithm that is used by Given Imaging), which makes it different to the algorithms described above and puts it closer to the category of algorithms aiming to detect abnormalities, as described in Section 3.3. 
The obvious conclusion regarding these methods must be that they are highly subjective. All research on this topic has to include particularly extensive clinical evaluations.

\subsection{Non-informative frame filtering}

Another idea for aiding capsule endoscopy video review involves removing non-informative frames from the video sequence. It was proposed by Vilarino et al. (2006) who presented an algorithm which detects areas in the WCE video comprising images completely obscured by intestinal fluids. Early detection of such regions is highly beneficial since they can be removed from the sequence, before it is presented to the clinician, resulting in a shortening of the reviewing time. Intestinal fluids appear as yellowish to brownish semi-opaque turbid liquids often containing air-bubbles as well as other artifacts. The authors point out that the most relevant feature of the intestinal fluids is the presence of small bubbles of different sizes and quasi-circular shapes. The algorithm is based on texture analysis performed using Gabor filter banks. In order to construct a filter bank, the authors used four different directions oriented at $0^{\circ}, 45^{\circ}, 90^{\circ}, 135^{\circ}$, arranged of four gaussian scales (sigma values of $1,2,4$ and 8), resulting in 16 filters in the bank. Frames that contained bubbles detected in more than $50 \%$ of the useful visualisation area were considered not valid for clinician analysis. The authors tested their algorithm on ten WCE videos in which the reduction in number of frames varied from 12 to $46 \%$ (mean $23 \%$ ). They plan to expand this work to consider postprandial cases, where the texture patterns are more irregular.

Bashar et al. (2009) propose a cascade method for none-informative frame filtering, which uses local color histogram to isolate highly contaminated non-bubbled frames $(\mathrm{HCN})$, and Gauss Laguerre Transform based multiresolution norm-1 energy feature to filter out significantly bubbled (SB) frames. Their algorithm comprises of two steps: 1) HCN frames are isolated by means of Support Vector Machine classifier and 2) SB frames are filtered out using automatic bubble segmentation followed by threshold operation, which leaves us with the remaining informative frames. In an experiment with 20,558 frames from the three CE videos they compare the performance of their new method with Gabor and discrete wavelet feature methods concluding that the former significantly outperforms the latter two.

\subsection{Intestinal contraction detection}

Intestinal contractions, which are of some relevance to clinicians constitute only around $1 \%$ of the WCE video. Vilarinao et al. (2005) propose using ROC curves with ensembles of classifiers to detect these contractions based on 34 low-level image features from 9 consecutive frames including: mean intensity; hole size; global contrast; correlations between three previous sequences; and the corresponding sequences averaged across the objects for the class "contractions" and the variance of intensity. Spyridonos et al. (2005) introduce a two stage contraction detection algorithm based on a Support Vector Classifier. Patterns of intestinal motility are encoded using a number of textural and morphological features, that include: $1^{\text {st }}$ order statistics (mean, standard deviation, skew, kurtosis estimated from the image histogram); $2^{\text {nd }}$ order statistics (energy, entropy, inertia, local homogeneity, cluster shade and cluster prominence); Local Binary Pattern histograms (radius $=2$, number of points =16); and morphological features of the intestinal lumen (blob area, blob shape (solidity), blob sharpness and blob deepness). The authors report $73.5 \%$ sensitivity, $98.8 \%$ specificity and a false alarm ratio of $60 \%$. The above figures were calculated from the test set comprising of 6 capsule videos. Detection of intestinal contractions is further pursued by Igual et al. (2007) who propose Eigenmotion-based Contraction Detection algorithm. First, the algorithm 
extracts the motion information of a set of contraction sequences in form of optical flow fields. Next, these motion data are transformed into the space of eigenmotions using Principal Component Analysis. Then, a subset of the eigenmotions is used to characterise the high dimensional optical flow information. Finally, this contraction representation is fed into the Relevance Vector Machine for classification. The authors show the results which suggest that motion information improves the contraction detection task. Moreover, they claim that the scheme they propose may speed up the analysis time.

\section{Conclusions}

In this chapter, a number of computer image analysis algorithms developed over the period of ten years and presented in the literature was described. The picture that emerges from this description is very positive as far as the future of this field is concerned. The number of papers published in the field increases fast and there is still plenty of valuable research to be conducted.

Of all the different areas described, arguably the most thoroughly studied was the area of topographic segmentation. The algorithms involved here were tested on full length videos, proved to perform well and were shown to reduce the video analysis time. Many of these algorithms could be used in their current form in some real viewing software applications. Regarding future research in this field, the main areas of focused research will involve finding new features, which would allow better discrimination between different tissue types. In particular interesting new prospects might be opened by the use of context features such as capsule location in 3-D, $\mathrm{pH}$ factor, temperature etc. Moreover, the bowel preparation procedure (emptying the colon), which may become the standard procedure prior capsule ingestion, may have the influence on tissue classification. On the one hand, it will uncover more colon and terminal ileum surface, but on the other hand, there will be less digestive remains in the colon, which are used in the colour and texture description. It remains to be seen, whether this will have a significant influence on intestine/colon classification.

Another major area of capsule research is bleeding detection. Here, a large number of clinical studies have been published, assessing the performance of Given Suspected Blood Indicator. The authors report different sensitivity and specificity figures, although, they generally agree that the current performance of SBI is insufficient, does not reduce the video viewing time and must be improved. Bleeding detection algorithms described in this chapter are very promising. There was at least one study, which contained a comprehensive comparison of the algorithm performance with the SBI carried out using the large set of full length videos. Moreover, the work described here demonstrated that it is possible to detect a large number of other abnormalities using this method and in this respect it may constitute a prelude to the solution for detection of much wider range of GI tract abnormalities.

As to detection of other pathological events, the computer vision research is still in its infancy. Neither Given nor Olympus offer any automatic tools capable of detecting abnormalities. The studies presented in Section 3.3 claim achieving different often very high accuracy figures on detection of certain abnormalities. However, the sizes of training and test sets used in these studies are insufficient to draw conclusions as to whether such systems can be used in a working application. None of the studies described in that section was tested on the full length videos and discussed the implications arising from the large number of false positive detections. According to the author, this is the most challenging field of research in capsule endoscopy image processing since detecting pathologies is the ultimate goal of 
reliable automatic tools. From the work presented in (Coimbra, Campos \& Cunha, 2006a), we can see that this task is very difficult since so far the features (in this particular study MPEG-7 visual descriptors) have not the sufficient discriminant power.

Another successful field of computer vision research is adapting the video play rate to the local video contents. Given Imaging Rapid Reader provides the first automatic tool - Quick View capable of shortening the viewing time. Given the previously mentioned difficulties regarding detection of single pathologies, this seems to be a reasonable way forward. Moreover, the authors of the publication presented in Section 3.5 claim their Quick View-like algorithm achieves reduction of video assessment time to 30 minutes, which is an impressive achievement.

Other algorithms presented in Section 3.6 and 3.7 attempt to detect either relevant frames, which should be kept for future viewing by the clinician or irrelevant frames, which may be discarded. Detecting both types could reduce video viewing time - in a particular algorithm, attempting to detect intestinal fluids, the authors report the mean reduction in a number of frames to be viewed by $23 \%$. Again, this is a very promising result and given what was said in the previous paragraphs about the difficulties of detecting single image pathologies, we strongly anticipate the growth of the number of algorithms, building on the same idea - instead of detecting particular pathologies, we would rather detect and discard irrelevant parts of the video (e.g. intestinal fluids) and again detect and focus attention of the clinician on particularly important frames (e.g. intestinal contractions).

There are still "unchartered territories" in the WCE image processing. All WCE computer vision research so far was focused on the early capsules designated for investigation of the small intestine. The more recent technologies enabling more detailed investigation of the oesophagus and the colon were not investigated in this context. In particular, the latter is expected to attract the significant interest from the image processing community.

There are a number of difficulties that WCE computer vision researchers face. The first problem results from the nature of the capsule video data and is particularly troublesome as far as event detection such as bleeding or abnormality is concerned. The problem is that although each video exam consists of around 50,000 images, these images include very few relevant abnormal events. Thus, it is difficult to build sufficiently general models from the large, one might think, set of say around 100 exams. The more specific the abnormality is the more serious this problem becomes. The author of this chapter has come across this problem when building blood colour distributions for bleeding detection. It is anticipated, however, that this problem will be even more difficult with regard to describing other pathologies since they are even rarer in the capsule videos than blood and take significantly larger number of forms. Having said that, it is not difficult to explain why abnormality detection, which is the ultimate goal of computer vision in WCE image analysis, have not achieved excellent results in the first ten years of capsule video research. This is even more apparent when we look at other areas of WCE computer vision research (such as topographic segmentation, intestinal fluid and contraction detection), where thanks to abundance of relevant data in each video, building general models was possible and consequently resulted in the significant progress in these areas.

The second problem, which will become more apparent in the forthcoming future is the lack of public databases of annotated WCE videos, which could be used for testing and comparing the performance of different algorithms as the number of systems addressing similar problems will increase. Building such a database is not an easy task, since the amount of clinicians work annotating hundreds of videos with respect to many types of events (not necessarily 
relevant to clinicians! e.g. intestinal fluids) is enormous, not to mention the size of the data and resulting server requirements. Moreover, the problem might become even more difficult when the amount of data significantly increases due to unavoidable increases in capsule image acquisition rate and image resolution.

Having said all that, there is no doubt that the computer vision research on WCE videos will become an important field of medical image processing and will gain much wider interest of the researchers in the coming years. With this field clearly maturing and the steady increase in the clinical usage of WCE, it is predicted a very bright future for clinical and computer research in this topic.

\section{References}

Arena, A., Boulougoura, M., Chowdrey, H. S., Dario, P., Harendt, C., Irion, K., Kodogiannis, V., Lenaerts, V. S. K. B., Menciassi, A., Puers, R., Scherjon, C. \& Turgis, D. (2005). Intracorporeal Videoprobe (IVP), Proceedings of the ICMCC 2005, The Hague, NL.

Argüellas-Arias, F., Caunedo, A., Romero, J., Sánchez, A., Rodrigues-Téllez, M., Pellicer, F. J., Argüellas-Martin, F. \& Herrerias, J. (2004). The Value of Capsule Endoscopy in Pediatric Patients with a Suspicion of Crohn's Disease, Endoscopy 36: 869-873.

Bashar, M., Mori, K., Suenaga, Y., Kitasaka, T. \& Mekada, Y. (2009). Detecting informative frames from wireless capsule endoscopic video using color and texture features, Proc. MICCAI, Springer, Lcture Notes in Computer Science (LNCS), Vol. 5242, pp. 603-11.

Bejakovic, S., Kumar, R., Dassopoulos, T., Mullin, G. \& Hager, G. (2009). Analysis of crohn's disease lesions in capsule endoscopy images, ICRA'09, pp. 2793-2798.

Berens, J., Mackiewicz, M. \& Bell, G. D. (2005). Stomach, Intestine and Colon tissue discriminators for Wireless Capsule Endoscopy (WCE) images., Proceedings of SPIE, Vol. 5747, pp. 283-290.

Berens, J., Mackiewicz, M., Bell, G. D. \& Jamieson, C. (2004). Can we detect when a Wireless Capsule Endoscope WCE leaves the stomach using computational colour techniques? A pilot study, Endoscopy (abstract) 36 (Suppl I): A76.

Berens, J., Mackiewicz, M., Fisher, M. \& Bell, G. D. (2005). Using colour distributions to discriminate tissues in Wireless Capsule Endoscopy images., Proceedings of Medical Image Understanding and Analyses 2005 Conference, Bristol, UK, pp. 107-110.

Bernstein, C. N. (2006). The epidemiology of inflammatory bowel disease in canada: A population-based study, The American Journal of Gastroenterology 101(7): 1559-68.

Bona, M. D., Bellumat, A., Cian, E., Valiante, F., Moschini, A. \& Bonai, M. D. (2006). Capsule endoscopy findings in patients with suspected crohn's disease and biochemical markers of inflammation, Digestive $\mathcal{E}$ Liver Disease 38(5): 331-5.

Boulougoura, M., Wadge, E., Kodogiannis, V. S. \& Chowdrey, H. S. (2004). Intelligent systems for computer-assisted clinical endoscopic image analyses, Proceedings of the Second International Conference Biomedical Engineering.

Brown, G., Fraser, C., Schofield, G., Taylor, S., Bartram, C., Phillips, R. \& Saunders, B. (2006). Video capsule endoscopy in peutz-jeghers syndrome: a blinded comparison with barium follow-through for detection of small-bowel polyps, Endoscopy 38(4): 385-90.

Caunedo-Alvarez, M. J.-S. J. R.-V. A. \& Herrerias-Gutierrez, J. M. (2006). Capsule endoscopy: a useful tool in portal hypertensive enteropathy, Gastrointestinal Endoscopy 64(1): 152.

Cobrin, G. M. et al. (2006). Increased diagnostic yield of small bowel tumors with capsule endoscopy, Cancer 107(1): 22-7. 
Coimbra, M., Campos, P. \& Cunha, J. P. S. (2005). Extracting clinical information from endoscopic capsules exams using MPEG-7 visual descriptors, 2nd European Workshop on the Integration of Knowledge Semantic and Digital Media Technologies, IEE.

Coimbra, M., Campos, P. \& Cunha, J. P. S. (2006a). MPEG-7 Visual Descriptors - Contributions for Automated Feature Extraction in Capsule Endoscopy, 16: 628-37.

Coimbra, M., Campos, P. \& Cunha, J. P. S. (2006b). Topographic segmentation and transit times estimation for endoscopic capsule exams, Proceedings of the IEEE International Conference on Acoustics, Speech, and Signal Processing, Vol. II, Toulouse, France, pp. 1164-7.

Coimbra, M., Kustra, J., Cunha, J. P. S. \& Campos, P. (2006). Combining color with spatial and temporal position of the endoscopic capsule for improved topographic classification and segmentation, Proceedings of the 1st International Conference on Semantic and Digital Media Technologies, Athens, Greece.

Culliford, A., Daly, J., Diamond, B., Rubin, M. \& Green, P. H. R. (2005). The value of wireless capsule endoscopy in patients with complicated celiac disease, Gastrointestinal Endoscopy 62(1): 55-61.

Dai, N., Gubler, C., Hengstler, P., Meyenberger, C. \& Bauerfeind, P. (2005). Improved capsule endoscopy after bowel preparation, Gastrointestinal Endoscopy 61(1): 28-31.

Dubcenco, E. et al. (2005). Capsule endoscopy findings in patients with established and suspected Crohn's disease: correlation with radiologic, endoscopic and histologic findings, Gastrointestinal Endoscopy 62: 538-544.

Duda, K., Zielinski, T., Duplaga, M., Grega, M. \& Leszczuk, M. (2007). Vq classification based on mpeg-7 visual descriptors for video endoscopic capsule localisation in the gastrointestinal tract, 15th European Signal Processing Conference (EUSIPCO 2007), Poznan, Poland.

Duda, K., Zielinski, T., Fraczek, R., Bulat, J. \& Duplaga, M. (2007). Localization of endoscopic capsule in the gi tract based on mpeg-7 visual descriptors, Imaging Systems and Techniques, 2007. IST '07. IEEE International Workshop on, pp. $1-4$.

Eisen, G. M., Eliakim, R., Zaman, A. et al. (2006). The accuracy of PillCam ESO Capsule Endoscopy Versus Conventional Upper Endoscopy for the Diagnosis of Esophageal Varices: A prospective Three-Center Pilot Study, Endoscopy 38(1).

Eliakim, R., Fireman, Z., Gralnek, I. M., Yassin, K., Waterman, M., Kopelman, Y., Lachter, J., Koslovsky, B. \& Adler, S. N. (2006). Evaluation of the PillCam Colon capsule in the detection of colonic pathology: results of the first multicenter, prospective, comparative study, Endoscopy 38(10).

Eliakim, R., Yassin, K., Shilomi, I., Suissa, A. \& Eisen, G. M. (2004). A novel diagnostic tool for detecting oesophagal pathology: the pillcam oesophagal video capsule, Aliment Pharmacol Ther 20: 1083-9.

Fry, L. C., Carey, E., Shiff, A. D., Heigh, R. I., Sharma, V. K., Post, J. K., Hentz, J. G., Fleischer, D. E. \& Leighton, J. A. (2006). The yield of capsule endoscopy in patients with abdominal pain or diarrhea, Endoscopy 38(5): 498-502.

Fuyono, I. (2005). Olympus finds market rival hard to swallow, Nature 438: 913.

Gao, M., Hu, C., Chen, Z., Zhang, H. \& Liu, S. (2010). Design and fabrication of a magnetic propulsion system for self-propelled capsule endoscope, Biomedical Engineering, IEEE Transactions on 57(12): $2891-2902$.

Gay, G., Delvaux, M. \& Rey, J. (2004). The Role of Video Capsule Endoscopy in the Diagnosis of Digestive Diseases: a Review of Current Possibilities, Endoscopy 36: 913-920. 
Hai, V., Echigo, T., Sagawa, R., Yagi, K., Schiba, M., Higuchi, K., Arakawa, T. \& Yagi, Y. (2006). Adaptive control of video display for diagnostic assistance by analysis of capsule endoscopic images, Proceedings of ICPR.

Hwang, S., Oh, J. \& Tang, S. J. (2006). Expectation Maximization based Bleeding Detection for Wireless Capsule Endoscopy (WCE) images., Proceedings of SPIE, Vol. 6144, pp. 577-587.

Iddan, G., Meron, G., Glukhovsky, A. \& Swain, P. (2000). Wireless capsule endoscopy, Nature 405: 725-9.

Igual, L., Seguí, S., Vitriàă, J., Azpiroz, F. \& Radeva, P. (2007). Eigenmotion-based detection of intestinal contractions, Lecture Notes in Computer Science 4673/2007: 293-300.

Innovative imaging probes for endoscopy (2005). European Innovations (4).

IntroMedic (n.d.).

URL: http://intromedic.com/

Kosa, G., Jakab, P., Jolesz, F. \& Hata, N. (2008). Swimming capsule endoscope using static and rf magnetic field of mri for propulsion, Robotics and Automation, 2008. ICRA 2008. IEEE International Conference on, pp. 2922 -2927.

Lee, J., Oh, J., Shah, S., Yuan, X. \& Tang, S. (2007). Automatic classification of digestive organs in wireless capsule endoscopy videos, Proceedings of the 2007 ACM symposium on Applied computing, SAC '07, ACM, New York, NY, USA, pp. 1041-1045.

URL: http://doi.acm.org/10.1145/1244002.1244230

Leighton, J. A., Sharma, V. K., Hentz, J. G., Musil, D., Malikowski, M. J., McWane, T. L. \& Fleischer, D. E. (2006). Capsule endoscopy versus push enteroscopy for evaluation of obscure gastrointestinal bleeding with 1-year outcomes, Digestive Diseases $\mathcal{E}$ Sciences 51(5): 891-9.

Lenaerts, B. \& Puers, R. (2006). An omnidirectional transcutaneous power link for capsule endoscopy, Proceedings of International Workshop on Wearable and Implantable Body Sensor Networks (BSN'06), pp. 46-9.

Li, B. \& Meng, M. Q.-H. (2009). Computer-based detection of bleeding and ulcer in wireless capsule endoscopy images by chromaticity moments, Computers in Biology and Medicine 39: 141-7.

Li, B., Meng, M. Q.-H. \& Lau, J. Y. (2011). Computer-aided small bowel tumor detection for capsule endoscopy, Artificial Intelligence in Medicine In Press, Corrected Proof.

Liao, Z., Li, F. \& Li, Z.-S. (2008). Clinical application of omom capsule endoscopy in china: a review of 1,068 cases, Gastrointest. Endosc. 67(5): AB265.

Mackiewicz, M., Berens, J. \& Fisher, M. (2008). Wireless capsule endoscopy colour video segmentation, 27(12): 1769-81.

Mackiewicz, M., Fisher, M. \& Jamieson, C. (2008). Bleeding detection in wireless capsule endoscopy using adaptive colour histogram model and support vector classification., Proceedings of SPIE, Vol. 6914.

Melmed, G. Y. \& Lo, S. K. (2005). Capsule Endoscopy: Practical Applications, Clinical Gastroenterology and Hepatology 3(5): 411-22.

Mishkin, D. S., Chuttani, R., Croffie, J. et al. (2006). ASGE Technology Status Report, Wireless Capsule Endoscopy, Gastrointestinal Endoscopy 63: 539-45.

Neu, B., Wettschureck, E. \& Rösch, T. (2003). Is esophagal Capsule Endoscopy Feasible? results of a pilot, Endoscopy 35: 957-961. 
Olympus News Release (2004). Development of capsule endoscopes and peripheral technologies for further expansion and progress in endoscope applications, on-line.

URL: http://www.olympus-global.com/en/news/2004b/nr041130capsle.cfm

Pennazio, M. (2004a). Capsule endoscopy, Endoscopy 37: 1073-8.

Pennazio, M. (2004b). Small-Bowell Endoscopy, Endoscopy 36: 32-41.

Quirini, M., III, R. W., A.Menciassi \& Dario, P. (2007). Design of a pill-sized 12-legged endoscopic capsule robot, Proc. of IEEE International Conference on Robotics and Automation, pp. 1856-62.

Qureshi, W. A. (2004). Current and future applications of the capsule camera, Nature 3: 447-50.

Ravens, A. F. \& Swain, P. (2002). The wireless capsule: new light in the darkness, Digestive Diseases 20: 127-33.

RF Systems (n.d.). Japan. URL: http://www.rfsystemlab.com/

Schoofs, N., Devière, J. \& Gossum, A. V. (2006). Evaluation of the PillCam Colon capsule in the detection of colonic pathology: results of the first multicenter, prospective, comparative study, Endoscopy 38(10).

Selby, W. (2004). Can clinical features predict the likelihood of finding abnormalities when using capsule endoscopy in patients with gi bleeding of obscure origin, Gastrointestinal Endoscopy 59: 782-7.

Sendoh, M., Ishiyama, K. \& Arai, K.-I. (2003). Fabrication of magnetic acurator for use in a capsule endoscope, 39(5): 3232-4.

Signorelli, C. et al. (2005). Sensitivity and Specificity of the Suspected Blood Identification System in Video Capsule Enteroscopy, Endoscopy 37: 1170-3.

Spyridonos, P., Vilarino, F., Vitrià, J. \& Radeva, P. (2005). Identification of intestinal motility events of capsule endoscopy video analysis., ACIVS, pp. 531-537.

Swain, P. (2005). Wireless capsule endoscopy and Crohn's disease, Gut 54: 323-326.

Szczypinski, P. \& Klepaczko, A. (2009). Selecting texture discriminative descriptors of capsule endpscopy images, Image and Signal Processing and Analysis, 2009. ISPA 2009. Proceedings of 6th International Symposium on, pp. $701-706$.

Szczypinski, P. M., Sririam, P. V. J., Sririam, R. D. \& Reddy, D. (2004). Model of deformable rings for aiding the wireless capsule endoscopy video interpretation and reporting, Proceedings of the International Conference on Computer Vision and Graphics, Warsaw, Poland, pp. 22-24.

Tang, S., Zanati, S., Dubcenco, E., Christodoulou, D., Cirocco, M., Kandel, G., Kortan, P., Haber, G. B. \& Marcon, N. E. (2003). Capsule endoscopy regional transit abnormality: a sign of underlying small bowell pathology, Gastrointestinal Endoscopy 58(4): 598-602.

Toennies, J., Tortora, G., Simi, M., Valdastri, P. \& III, R. W. (2010). Swallowable medical devices for diagnosis and surgery: the state of the art, Proc. IMechE Vol. 224 Part C: J. Mechanical Engineering Science, Vol. 224, pp. 1397-1414.

Turgis, D. \& Puers, R. (2004). Image compression in video transmission for capsule endoscopy, Eurosensors XVIII.

Urbain, D., Looze, D. D., Demedts, I., Louis, E., Dewit, O., Macken, E. \& Gossum, A. V. (2006). Video capsule endoscopy in small-bowel malignancy: a multicenter belgian study, Endoscopy 38(4): 408-11.

van Tuyl, S. A. C., van Noorden, J. T., Timmer, R., Stolk, M. F. J., Kuipers, E. J. \& Taal, B. G. (2006). Detection of small-bowel neuroendocrine tumors by video capsule endoscopy, Gastrointestinal Endoscopy 64(1): 66-72. 
Viazis, N. et al. (2005). Inpact of capsule endoscopy in obscure small-bowel bleeding: defining strict diagnostic criteria for a favorable outcome, Gastrointestinal Endoscopy 62: 717-722.

Vilarinao, F., Kuncheva, L. I. \& Radeva, P. (2005). ROC curves and video analysis optimization in intestinal capsule endoscopy, Pattern Recognition Letters, Special Issue on ROC Analysis .

Vilarino, F., Spyridonos, P., Puyol, O., Vitrià, J. \& Radeva, P. (2006). Automatic detection of intestinal juices in wireless capsule video endoscopy, Proceedings of ICPR. 


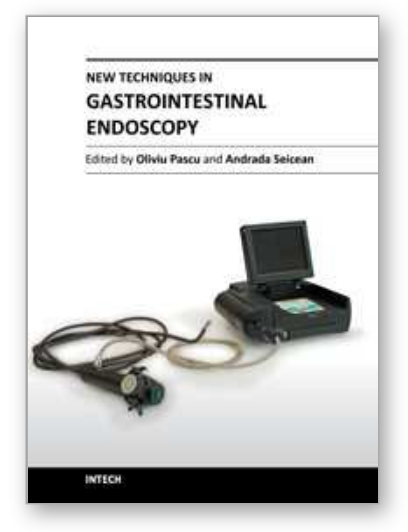

\author{
New Techniques in Gastrointestinal Endoscopy \\ Edited by Prof. Oliviu Pascu
}

ISBN 978-953-307-777-2

Hard cover, 310 pages

Publisher InTech

Published online 30, September, 2011

Published in print edition September, 2011

As result of progress, endoscopy has became more complex, using more sophisticated devices and has claimed a special form. In this moment, the gastroenterologist performing endoscopy has to be an expert in macroscopic view of the lesions in the gut, with good skills for using standard endoscopes, with good experience in ultrasound (for performing endoscopic ultrasound), with pathology experience for confocal examination. It is compulsory to get experience and to have patience and attention for the follow-up of thousands of images transmitted during capsule endoscopy or to have knowledge in physics necessary for autofluorescence imaging endoscopy. Therefore, the idea of an endoscopist has changed. Examinations mentioned need a special formation, a superior level of instruction, accessible to those who have already gained enough experience in basic diagnostic endoscopy. This is the reason for what these new issues of endoscopy are presented in this book of New techniques in Gastrointestinal Endoscopy.

\title{
How to reference
}

In order to correctly reference this scholarly work, feel free to copy and paste the following:

Michał Mackiewicz (2011). Capsule Endoscopy - State of the Technology and Computer Vision Tools After the First Decade, New Techniques in Gastrointestinal Endoscopy, Prof. Oliviu Pascu (Ed.), ISBN: 978-953-307777-2, InTech, Available from: http://www.intechopen.com/books/new-techniques-in-gastrointestinalendoscopy/capsule-endoscopy-state-of-the-technology-and-computer-vision-tools-after-the-first-decade

\section{INTECH}

open science | open minds

\author{
InTech Europe \\ University Campus STeP Ri \\ Slavka Krautzeka 83/A \\ 51000 Rijeka, Croatia \\ Phone: +385 (51) 770447 \\ Fax: +385 (51) 686166 \\ www.intechopen.com
}

\author{
InTech China \\ Unit 405, Office Block, Hotel Equatorial Shanghai \\ No.65, Yan An Road (West), Shanghai, 200040, China \\ 中国上海市延安西路65号上海国际贵都大饭店办公楼 405 单元 \\ Phone: +86-21-62489820 \\ Fax: $+86-21-62489821$
}


(C) 2011 The Author(s). Licensee IntechOpen. This chapter is distributed under the terms of the Creative Commons Attribution-NonCommercialShareAlike-3.0 License, which permits use, distribution and reproduction for non-commercial purposes, provided the original is properly cited and derivative works building on this content are distributed under the same license. 